\title{
BURNING RATE OF LIQUID FUEL ON CARPET (POROUS MEDIA)
}

\author{
Tingguang Ma, Stephen M. Olenick, Michael S. Klassen, Richard J. Roby \\ Combustion Science \& Engineering, Inc. \\ 8940 Old Annapolis Road - Suite L \\ Columbia, Maryland 21045 \\ USA \\ and
}

Jose L. Torero

School of Civil and Environmental Engineering

The University of Edinburgh

Edinburgh, EH9 3JN

United Kingdom

\author{
Correspondence: \\ Tingguang Ma \\ tgma@wam.umd.edu \\ Current address: \\ 0151 Eng. Bldg, \\ Fire Protection Eng. Dept., \\ University of Maryland, College Park, MD20770 \\ Office number: 301-405-3999
}


Abstract. The occurrence of a liquid fuel burning on carpet has been involved in many incendiary and accidental fires. While the research on a liquid fuel fire on carpet is still limited, much work on porous media has been performed using sand or glass beads soaked with liquid fuel. In this study, a heat and mass transfer theory was first developed to analyze the burning process of liquid on carpet, and then several small-scale tests were performed to validate the theory. This analysis is valid for pool fires intermediate in size (5-20 cm. in diameter). The experimental apparatus consisted of a circular pan (105mm) and a load cell. Varying amounts of fuels (heptane, kerosene and methanol) were spilled onto the carpet, which was allowed to burn in a quiescent environment. It was found that due to the different controlling mechanisms, the liquid burning rate could be less or more than that of a similarly spilled free-burning pool fire. For the worst-case scenario in fires, the maximum enhancement of the burning rate due to the porous media is predictable through the physical properties of the fuel. This analysis is valid for both combustion and evaporation. Several similar results in the scientific literature are analyzed to further describe the trend. This work explains the role of carpet in liquid pool fires and also helps to explain special risks related to the presence of carpet involved in arsons and will be useful in reconstruction of the early development of an incendiary or accidental fire.

Key words: Liquid burning, porous media, carpet, burning rate, fire investigation, arsons, fire reconstruction 


\section{Introduction}

The role of carpets in fire has attracted fire research interests since the 1960's and a number of test procedures have been developed to assess the flammability of carpets involved in fire [1]. Typically these test procedures address ignition and flame spread on carpets. A scenario of great practical importance is that of an incendiary fire with an ignitable liquid burning on the carpet. In this particular case, the flammability of the carpet is not necessarily the issue of main importance, but instead the significance is the role that the carpet has on the burning of the liquid fuel. This combination can cause a greater hazard than the fuel or the carpet alone [2].

For liquids burning on carpets, the fire will behave like a pool fire, due to the low fire point of liquid fuel, though the porous medium will affect the magnitude of the different transfer processes which occur during burning. Joulain [3] recently reviewed the pool fire literature, and therefore a similar review will not be included in this paper. Of more immediate relevance to this work are those pool fire studies where the different mechanisms of heat transfer have been analyzed. Based on the extensive experimental results of Blinov and Khudiakov [4], Hottel [5] analyzed the independent contribution of three modes of heat feedback from the flame. Spalding [6] neglected radiative heat exchange and modeled liquid fuel burning by introducing the concept of the mass transfer number ("B" number). The "B" number included a further major simplification that eliminated in-depth heat transfer by assuming that the fuel is all at a constant temperature. During burning, changes in heat transfer are reflected in the burning rate. Burgess et al. [7] studied the burning of liquid fuel in open trays and discussed the 
parameters affecting the burning rate. Akita [8] and Klassen et al. [9] analyzed the heat transfer modes for small pool fires. A global model for predicting the burning rates of liquid pool fires is generalized by Hamins et al. [10]. The effect of energy accumulation within the fuel and its effect on the burning rate were studied by Hayasaka [11] for small pool fires.

Much research work has been conducted on a liquid fuel burning by using different forms of porous media; nevertheless, none of these studies have concentrated on the actual effects of the substrate on the burning characteristics. Ishida [12], Takeno and Hirano [13], Takeuchi et al. [14] used glass beads, and Suzuki and Hirano [15] used sand to study the flame spread behavior of fuel on porous media. The capillary rise effect was found to be important in determining the fuel consumption rate. Blackshear and Kanury [16] state that the fuel consumption rate in wick burning was nearly the same as that in comparable experiments in which liquid fuels burn in horizontal pans. Tao and Kaviany [17] studied the burning rate of a liquid fuel supplied through a ceramic wick. Wentworth and Torero [18] studied the ignition and mass-burning of insulation material soaked with liquid fuel.

The present study attempts to provide a phenomenological description of the effect of carpet on the burning characteristics of liquid fuels. Several exploratory tests were done to validate the theory. Precise experiments were difficult to perform in many ways. First, carpet is different from the porous media used in the related studies in many ways. Carpet is not of uniform composition since it has basically two parts, well-structured fibers 
(usually made of nylon, polyester, olefin, wool, etc.) and water resistant backing (usually made of polyurethane plastic compounds). Therefore, it is difficult to characterize carpets using a single parameter such as diameter or porosity. Second, the role of fuel type can be quite important in these fires. The variations caused by fuel type, amount, and application mode (method of spill) are significant [19]. Finally, when liquid fuel burns on carpet fibers, the evaporation surface is no longer two-dimensional, as is found in most free-burning pool fires. The extended burning surface due to the carpet fibers makes the problem more complex. Here, a theory for liquid fuel burning on carpet is proposed first, then the results of a series of small-scale tests to validate the theory are described, and finally experimental results from other researchers are analyzed to validate different aspects of the theory. The possible uses of the theory are also discussed.

\section{Analysis of liquid burning on carpets}

\subsection{Surface Heat Balance}

The evaporation and burning process of liquid fuel is controlled by both mass transfer and heat transfer processes. Using Spalding's [6] B number mass transfer theory, the burning rate can be described by means of a simple expression

$$
\dot{m}^{\prime \prime}=\rho_{\text {sat }} u_{\text {sat }}=\frac{\rho D}{\delta} \ln (1+B),
$$

where $\mathrm{B}$ is the mass-burning number, $\delta$ a characteristic thermal penetration depth and $\mathrm{D}$ the mass diffusivity of the fuel vapor in air. The mass-burning rate, $\dot{m}^{\prime \prime}$, can be in turn split into the product of the saturation density, $\rho_{\text {sat }}$, and the surface regression velocity, $\mathrm{u}_{\text {sat }}$. The presence of the porous media implies in-depth heat transfer; therefore, the mass transfer number must be modified to incorporate this, leading to: 


$$
B=\frac{s y_{o, \infty}+y_{s a t}}{1-y_{s a t}}=\frac{c_{p}\left(T_{\infty}-T_{s a t}\right)+s y_{o, \infty} Q}{L_{v}+Q_{T L}},
$$

where $L_{v}$ is the latent heat of evaporation, $Q_{T L}=A \cdot\left(\dot{q}_{i n}^{\prime \prime}+\dot{q}_{\text {out }}^{\prime \prime}\right) / \dot{m}^{\prime \prime}$ is the total energy loss term which is the total energy received minus the portion needed for evaporation, and $\mathrm{A}$ is the effective diffusion surface area $A=\pi D^{2} / 4$. The mass transfer boundary condition also allows the expression of the mass transfer number as a function of the surface fuel concentration, where $\mathrm{s}$ is the stoichiometric fuel-air ratio and $y_{o, \infty}$ is the mass fraction of oxygen in the air stream. From equation (2), we can see that the term $\dot{q}_{I n}^{\prime \prime}$ is important in determining the mass-burning rate, which requires detailed heat transfer analysis.

A schematic diagram of the heat balance in a liquid fuel pool fire is shown in Figure 1. The heat balance at the burning surface is presented in equation (3).

$$
\begin{aligned}
& \dot{q}_{T}^{\prime \prime}=\dot{q}_{F}^{\prime \prime}+\dot{q}_{C d}^{\prime \prime}=\left(\dot{q}_{R}^{\prime \prime}+\dot{q}_{C V}^{\prime \prime}\right)+\dot{q}_{C d}^{\prime \prime}=\dot{q}_{V}^{\prime \prime}+\dot{q}_{I n}^{\prime \prime}+\dot{q}_{\text {Out }}^{\prime \prime} \\
& =\dot{q}_{V}^{\prime \prime}+\left(\dot{q}_{I n, C V}^{\prime \prime}+\dot{q}_{I n, C d}^{\prime \prime}+\dot{q}_{\text {Loss }}^{\prime \prime}\right)+\left(\dot{q}_{S, R R}^{\prime \prime}+\dot{q}_{R}^{\prime \prime}\right)
\end{aligned}
$$

The total heat received at the surface, $\dot{q}_{T}^{\prime \prime}$, corresponds to the summation of the energy feedback from the flame, $\dot{q}_{F}^{\prime \prime}$, and any conduction coming from the pan rim $\dot{q}_{C d}^{\prime \prime}$. The flame contribution, $\dot{q}_{F}^{\prime \prime}$, can be subsequently divided into radiative, $\dot{q}_{R}^{\prime \prime}$, and convective, $\dot{q}_{C V}^{\prime \prime}$, components. A portion of $\dot{q}_{T}^{\prime \prime}$ is used to evaporate the fuel, $\dot{q}_{V}^{\prime \prime}$, part is transferred in-depth, $\dot{q}_{I n}^{\prime \prime}$, and the rest is lost, $\dot{q}_{\text {Out }}^{\prime \prime}$. The magnitude of $\dot{q}_{V}^{\prime \prime}$ determines the massburning rate, so it is the main focus of our study. The heat losses, $\dot{q}_{\text {Out }}^{\prime \prime}$, can be in turn 
split into surface reflection, $\dot{q}_{R}^{\prime \prime}$, and surface re-radiation, $\dot{q}_{S, R R}^{\prime \prime}$; and the in-depth heat transfer, $\dot{q}_{I n}^{\prime \prime}$, can include the conductive component, $\dot{q}_{I n, C D}^{\prime \prime}$, convective, $\dot{q}_{I n, C v}^{\prime \prime}$, (in case there is fluid motion within the pan) and a term for side and bottom losses to the pan, $\dot{q}_{\text {Loss }}^{\prime \prime}$. It is important to clarify that the term $\dot{\mathrm{q}}_{\text {Loss }}^{\prime \prime}$ is actually not a surface loss but a lumped approximation that accounts for the impact that heat losses from the substrate might have on the surface boundary condition.

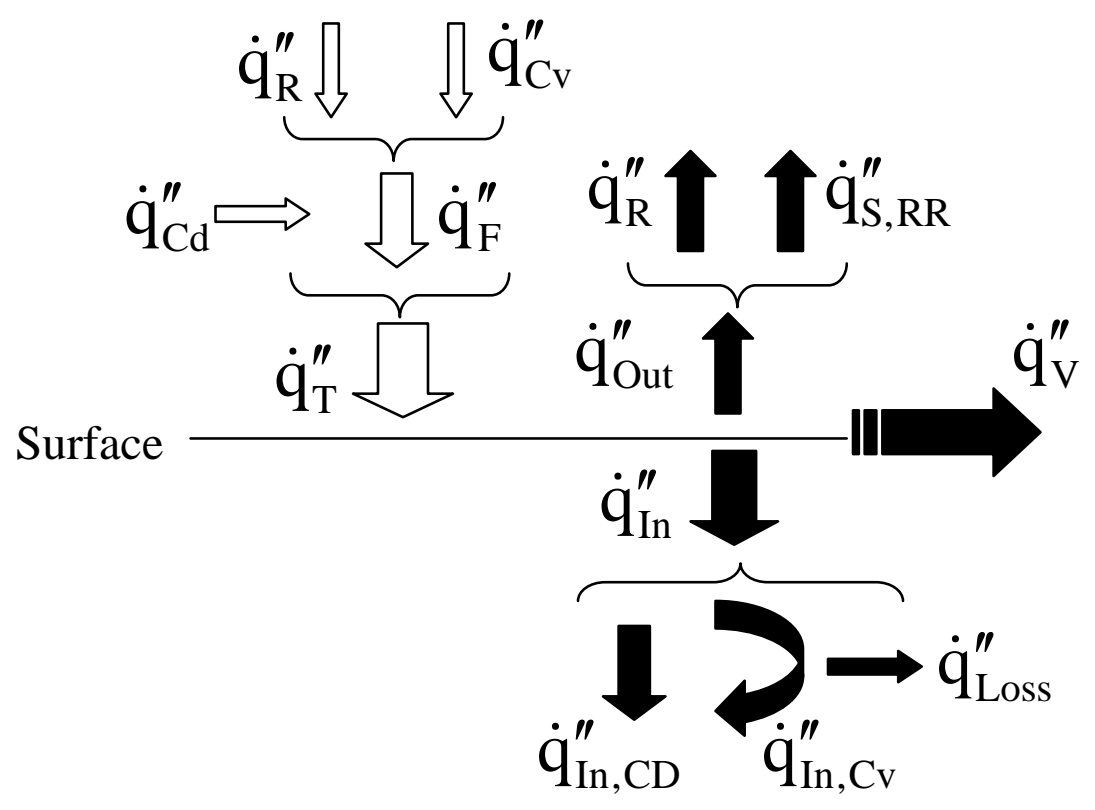

Figure 1. Schematic diagram of the energy balance for a liquid pool fire

As shown in previous sections, when a flame is established on a carpet soaked with fuel, the relative contribution of the loss terms will be different than for a free burning pool fire. The total energy balance at the surface will thus be modified, leading to a different mass-burning rate. It is important to establish in detail how each term of equation (1) will be modified. Throughout this initial analysis, for simplification, it will be assumed that the heat feedback from the flame, $\dot{q}_{F}^{\prime \prime}$, is unaffected by the carpet. 
Surface reflection can be considered a fraction of $\dot{q}_{T}^{\prime \prime}$ (i.e. reflectivity) and re-radiation should remain unaffected while the fuel is still close to the surface. It is difficult to evaluate the evolution of the surface re-radiation term as the saturation level varies and, eventually, it will be expected to increase once the fuel is completely gone from the surface and the carpet surface temperature increases beyond the boiling point of the fuel. Nevertheless, with enough fuel supply, the surface temperature will remain low (due to successive cooling from the evaporation of fuel) and the variation could be neglected, thus $\dot{q}_{S, R R}^{\prime \prime}$ could be considered a small constant fraction. Please note that when the liquid fuel is gone, not all carpet fiber behave the same. Wool chars and ashes, polypropylene and acrylics melt, nylon does both. The fiber materials also vary in the amount of soot they produce. If there is fuel surrounding the fiber, this behavior is not important.

An important change when the carpet is introduced is that of $\dot{\mathrm{q}}_{\mathrm{In}}^{\prime \prime}$, since it is changed in both the convection and the conduction terms. The liquid in the carpet is not free to circulate; therefore, $\dot{q}_{I n, C v}^{\prime \prime}$ is diminished. The long and dense fibers block the surface reflection and re-radiation from the liquid. The dominant heat transfer in carpet soaked with fuel is heat conduction. Here the poor conductivity of the carpet may be the source of the heat loss reduction. As seen in the estimation from Table 1, the thermal conductivity of carpets is approximately 1 order of magnitude lower than liquid fuel, and two orders of magnitude lower than sand. Furthermore, when the liquid fuel is below the carpet tip, the capillary effect supplies the fuel along the carpet fibers. This has two effects, the liquid evaporation happens only at the surface of the fiber, and the supply of 
the fuel counter-acts the heat conduction into the fiber. The global effect is that the liquid reaches the boiling point in a very thin layer on the surface of the fibers. Therefore, the temperature difference (from the ambient temperature to the fire point of the fuel) for a certain fuel is almost always the same, and the main in-depth heat transfer term, $\dot{q}_{I n, C D}^{\prime \prime}$, is significantly blocked, if it is still measurable. The extended fiber surface also reduces the surface re-radiation and reflection. The poor conductivity of liquid in the carpet also lowers the loss to the environment, $\dot{q}_{\text {Loss }}^{\prime \prime}$. So the overall value of $\dot{q}_{\text {In }}^{\prime \prime}$ is thus significantly reduced.

\begin{tabular}{|l|l|}
\hline Material & $\begin{array}{l}\text { Conductivity } \\
\text { (@ 300K), W/m-K }\end{array}$ \\
\hline Water & 0.614 \\
\hline Polystyrene & 0.11 \\
\hline Nylon 66 & 0.4 \\
\hline Sandstone & 1.83 \\
\hline Tin, pure & 65.0 \\
\hline
\end{tabular}

Table 1. Approximate material conductivities for various materials [22].

In summary, $\dot{\mathrm{q}}_{\mathrm{In}}^{\prime \prime}$ is reduced while all other terms seem unaffected by the carpet. Therefore, more heat is reserved to evaporate the fuel on the burning surface, increasing the evaporating and mass-burning rate.

\subsection{Mechanism of Fuel Transport}

Though carpets may appear in different forms and materials, their basic components, fibers and textiles, are almost the same in the manner they are constructed, and they can be treated as porous media. In the heat transfer analysis, this translates into the inhibition of fluid motion and the change in the overall thermal properties. From a mass transfer perspective, the presence of a porous medium implies further effects. The interface 
between the liquid and the solid phases is not planar and the distribution of the phases on this interface is very complex and depends on the surface saturation, the topology of the carpet, the ability of the carpet to absorb fuel, and the burning history. When the ignitable liquid is spilled on carpet, the gaseous phase moves due to the concentration and the total gas-phase pressure gradients. The liquid phase, in addition to the saturation gradient, can undergo motion due to the gradient of the surface-tension, buoyancy, wind-shear, solidfluid surface forces, or a combination of these forces.

When the amount of fuel is not sufficient to saturate the carpet, the space between the fibers is not completely filled. The porous media extends the surface area between the liquid and gas phases, but the effective diffusion area is almost the same. The fuel vapor concentration level at the carpet surface is lower compared with that at the surface of a free-burning liquid pool, which is close to unity. Mass transfer is therefore limited due to this partial saturation.
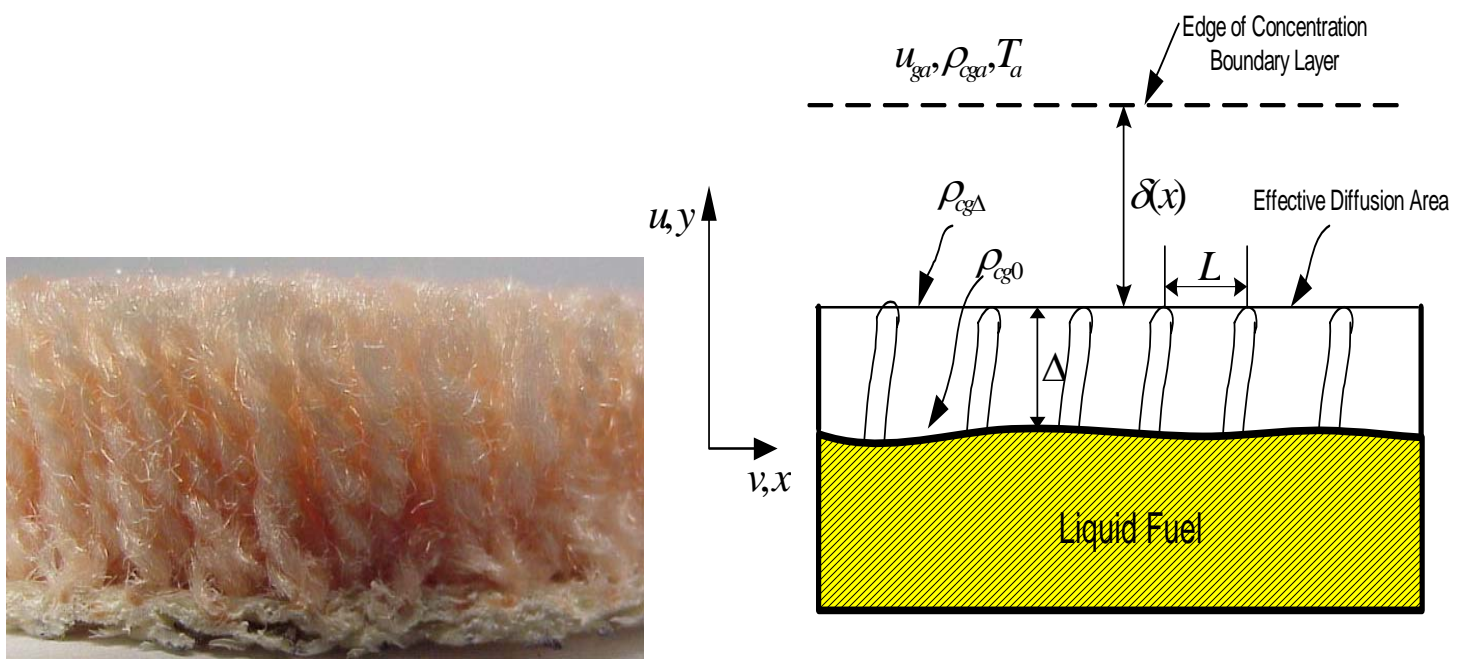

Figure 2. Structure of carpet (cut pile, $1.4 \mathrm{~cm}$ in thickness) and a schematic showing variables important for mass transfer of liquid burning on carpet 
To study the role of fuel saturation, Tao and Kaviany [17] used a one-dimensional treatment that lead to the following expression for the evaporation rate on carpet:

$$
\dot{m}^{\prime \prime}=\frac{\rho_{g} D_{m}}{\delta} \ln \left(\frac{\rho_{g}-\rho_{c g a}}{\rho_{g}-\rho_{c g \Delta}}\right)
$$

where $D_{m}$ is the mass diffusion coefficient. Details of the variables for this equation are discussed below and shown in Figure 2. $\rho_{c g a}, \rho_{c g \Delta}$, and $\rho_{c g 0}$ are vapor density at ambient, carpet surface, pool surface respectively. $\rho_{g}$ is the vapor density at any arbitrary location within the boundary layer.

If the liquid level is above the fiber tip, or if the saturation level is unity, the evaporation rate will be

$$
\dot{m}^{\prime \prime}=\frac{\rho_{g} D_{m}}{\delta} \ln \left(\frac{\rho_{g}-\rho_{c g a}}{\rho_{g}-\rho_{c g 0}}\right)
$$

This treatment corresponds to the surface of the porous medium soaked in fuel over which a boundary layer is established and where mass transfer occurs between the liquid and the air. The thickness of the boundary layer $(\delta)$ is determined by the flow over the substrate surface, therefore the burning rate increases with an increase of the external flow. For a given carpet type, $\delta$ will be assumed constant. The density of the fuel on the liquid surface is $\rho_{c g 0}$ and the density of the fuel vapor on the effective diffusion surface is $\rho_{c g \Delta}$. The density of the fuel present at the interface is $\rho_{\mathrm{f}, \mathrm{i}}$ which shows that a decrease in the saturation level (which will decrease $\rho_{c g \Delta}$ ) leads to a decrease in the evaporation rate. 
The structure of the carpet could have an effect on the extent and characteristics of the different mass-burning rate stages. Tao and Kaviany [17] showed, for the evaporation of ethanol into air, that at a transitional value of $\delta / L<30$ ( $\mathrm{L}$ is the distance between solid surfaces; i.e. carpet fibers), the effect of the saturation level on the mass-burning begins to become significant. This above analysis is generally valid for most dry porous media, such as ceramics or glass beads.

For the complex fibers used in carpet, the lower concentration level is not significant, as the capillary effect will supply the fuel to the fiber tip continuously, which counteracts the diffusion limitation. Only in the later stage of burning, when the liquid level is very low and the capillary effect is not sufficient to supply the fuel to the fiber tip, will the mass transfer limiting effect become significant. This effect is evident from the vastly different magnitudes of the densities at the liquid surface and in the vapor space and their role in the mass-burning rates, since

$$
\rho_{c g a}<\rho_{c g \Delta} \leq \rho_{c g 0} .
$$

The early and steady burning stages are not affected by this phenomenon.

Capillary effects can also explain this phenomenon. The lower level of liquid in the carpet translates into a longer distance for the carpet fiber to transport fuel. For fires with a sufficient fuel supply, capillary action supplies the fuel. When the capillary effect is not strong enough to supply the fuel, the temperature of the fibers will increase as there is not enough cooling effect from the liquid fuel. The burning rate becomes lower accordingly until there is no remaining liquid fuel supply and the carpet fibers provide fuel from the 
fire. But this effect is significant only at a later stage, and is difficult to quantify. Therefore, there is no clear theory for predicting the mass-burning rate based on capillary effects alone. In-depth fuel transfer is a very complex phenomenon under this environment, some research work is still in need to fully understand this.

\subsection{Maximum Burning Rate Ratio}

The final element that requires detailed attention is the possibility of establishing a maximum burning rate for fuel saturated carpet. It has been shown that the presence of carpet tends initially to increase the burning rate and then decrease the burning rate in the later stage. An analysis of the heat and mass transfer at the interface has served to clarify the relative importance of the physical parameters involved. The uncertainty in the structure and properties of the carpet as well as the assumptions of the analysis make it difficult for equations (1) and (4) to be used for quantitative predictions of the burning rate. In the face of this uncertainty, it is essential for a fire investigator to be able to establish a threshold level that cannot be exceeded by the mass-burning rate. Since engineers and fire investigators often care about the maximum risk and worst case scenario, the maximum burning rate will be of primary interest. For this purpose, the concept of a Maximum Burning Rate Ratio (MBRR), first introduced by Hayasaka [11], will be applied here.

Hayasaka [11] noticed an interesting transient phenomenon in liquid pool fires. After the ignition of the fuel, the initial burning rate will generally increase until a steady burning

rate is achieved. Hayasaka measured the temperature in the liquid pool, and found that 
the boiling zone in the liquid fuel controls the temperature profile. Initially, the energy absorbed by the fuel will be used not only for evaporation, but also is partly lost due to in-depth heat conduction to the fuel. When a boiling zone is formed at the surface, the temperature difference between this zone and the deep pool is constant, so the heat loss is steady and a fixed fraction of the feedback. This term is minimally affected by the energy from the flame. From the viewpoint of the energy balance, the energy received is used totally for evaporation and the conduction loss is translated to a small and fixed increase of the heat of evaporation. Hayasaka [11] used the MBRR to describe this enhancement due to the blocking of the heat conduction into the pool. The MBRR is defined as the ratio of the total energy required to heat the fuel from its initial temperature to the boiling temperature (maximum possible energy needed, i.e. minimum burning rate) over the pure evaporation energy (minimum energy needed, i.e. therefore maximum burning rate). In a situation with fuel saturating carpet, a boiling zone layer still exists, but it is very thin, as the capillary induced velocity counters the heat conduction effect. The global effect is that heat conduction is blocked, so we can use MBRR to describe either phenomenon.

Assuming the energy feedback from the flame is constant, the energy requirement for evaporation has the following relationship with the mass-burning rate:

$$
M B R R=\frac{\Delta H_{e}+c_{p}\left(T_{b}-T_{0}\right)}{\Delta H_{e}} \propto \frac{\dot{m}_{f, \max }^{\prime \prime}}{\dot{m}_{f, \text { min }}^{\prime \prime}} .
$$

Hayasaka [11] made theoretical MBRR predictions for a number of different fuels together with some experimental values used for validation. The data presenting this information is reproduced from Hayasaka [11] in Figure 3. Most of the data points are 
calculated from the physical properties of the fuel (using equation 7). Experimental data from Hayasaka was obtained by comparing the initial burning rate of a pool fire (minimum burning rate) with the final burning rate (maximum burning rate). Once the boiling zone has appeared in the deep fuel pool, the heat loss (for preheating fuel) measures almost zero, so the mass-burning rate increases by a certain ratio.

In this study, the carpet fibers serve the same role as the boiling zone; they block the energy transfer to the deep pool. The carpet fiber and/or the capillary motion blocked the heat from being lost to the incoming fresh fuel. Though not measurable, we still can assume that a boiling zone exists on the surface of fibers, around which the fuel evaporates. The heat blocking mechanisms are different, but the resulting phenomena are same; therefore, the concept of MBRR from Hayasaka [11] applies to the problem of liquid fuel burning on carpet.

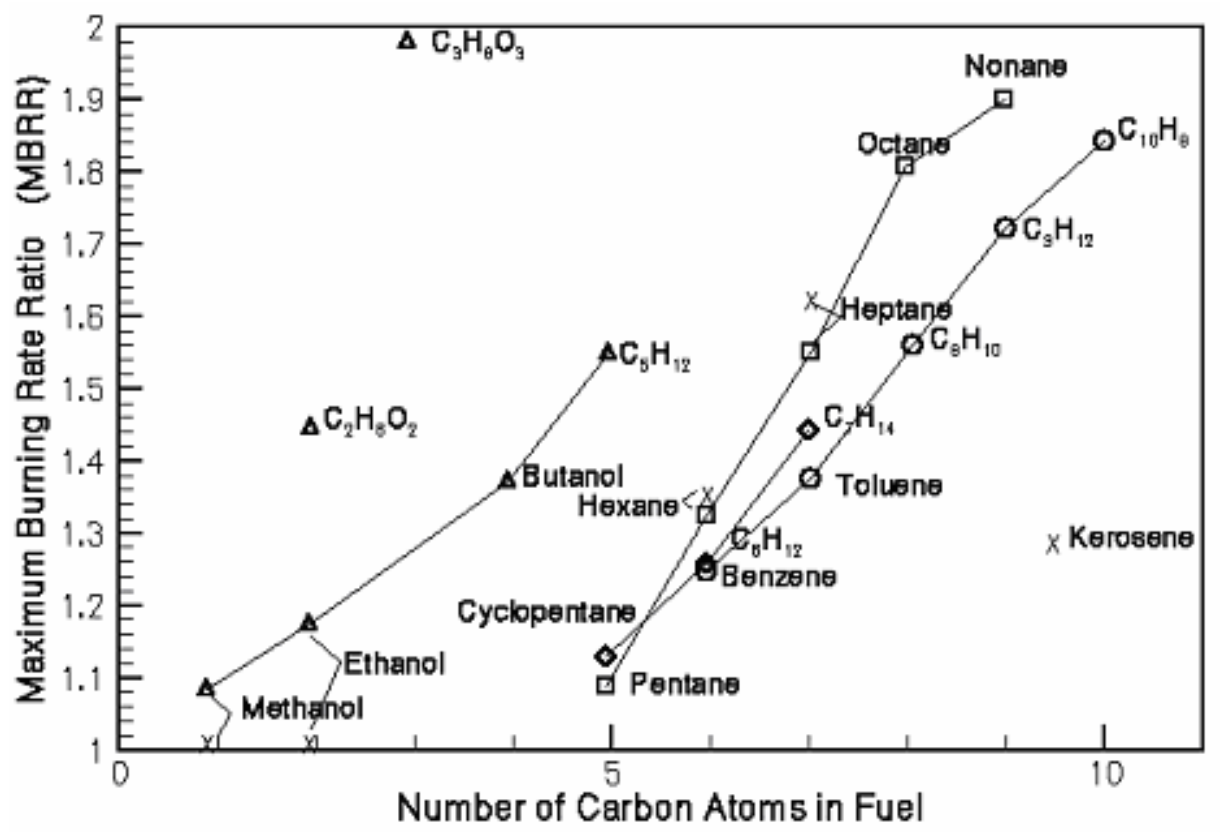


Figure 3. The theoretical and experimental MBRR of various fuels. The squares, triangles and diamonds are calculations from the physical properties of the fuels. The crossed points are experimental data. Reproduced from Hayasaka [11].

In summary, the heat loss term for preheating seems to be the main reason for enhanced burning rate of liquid burning on carpet. In this study, several fuels and different media are applied to find the effect of the boundary on the mass-burning rate. Some pre-burning evaporation tests are also performed to show their similarity to the combustion process. The carpet fibers are shown to block the in depth heat transfer, thus increasing the massburning rate.

\section{Validation Experiments}

As discussed earlier, several liquid fuels burning on carpets are tested to show the role of carpet on the liquid burning behavior. Some other porous media are also tested as a reference to show the special features of the carpet. Different type and variable amount of fuel were used to show the generality of the above theory.

\subsection{Experimental Apparatus}

The experimental apparatus consisted of a tin pan with a diameter of $105 \mathrm{~mm}$ placed on top of a load cell. The pan has a wall of $0.5 \mathrm{~mm}$ in thickness and is $14 \mathrm{~mm}$ in depth. A load cell was used to record the fuel mass loss during the experiment. The load cell has a range of 500 grams and a precision of 0.01 grams. A piece of paper cardboard $1 \mathrm{~mm}$ thick, covered by aluminum foil, was used as the insulation material between the pan and the load cell. The entire experimental setup was placed in a 2.2 x 2.6 x $2.4 \mathrm{~m}$ room with 
vertical ventilation through the ceiling. The room was isolated once the fire was ignited to minimize disturbances.

A common residential carpet type was used in this study, consisting of a Nylon $14 \mathrm{~mm}$ thick cut-pile and a backing material made of flexible polyurethane foam. A single carpet was chosen to minimize the number of variables but it is recognized that the physical characteristics of the carpet and backing material could potentially affect the results. The variants related to the nature of the carpet were considered to only have a quantitative impact on the results but will not affect the main conclusions reached through this study. Reference tests were conducted using sand as the porous medium to provide an estimate of the effect of the thermal properties of the porous medium on the burning characteristics. The sand chosen was Quacksand ${ }^{\mathrm{TM}}$ with a mean particle diameter of 0.8 $\mathrm{mm}$. On occasion, water was used as an insulation material. Without the water, the heat loss to the pan wall can provide energy feedback to the fuel, further complicating the problem.

Three fuels, heptane, kerosene, and methanol, with significantly different thermal properties were tested. Heptane is a volatile fuel, while kerosene is a combustible fuel with a high vapor pressure (i.e. evaporation rate is very slow at typical conditions). Methanol has a very significant latent heat of evaporation. The physical and chemical properties of the fuels are summarized in Table 2.

\begin{tabular}{|c|c|c|c|c|c|c|c|c|}
\hline Fuel Type & $\begin{array}{c}\text { Heat of } \\
\text { Evaporation }\end{array}$ & $\begin{array}{c}\text { Heat of } \\
\text { Combustion }\end{array}$ & $\begin{array}{c}\text { Boiling } \\
\text { Point }\end{array}$ & $\begin{array}{c}\text { Relative } \\
\text { Evaporation Rate }\end{array}$ & $\begin{array}{c}\text { Flash } \\
\text { point }\end{array}$ & Density & $\begin{array}{c}\text { Soot } \\
\text { Yield }\end{array}$ & Ref. \\
\hline & $\mathrm{kJ} / \mathrm{kg}$ & $\mathrm{MJ} / \mathrm{kg}$ & ${ }^{\circ} \mathrm{C}$ & n-butyl acetate=1 & ${ }^{\circ} \mathrm{C}$ & $\mathrm{kg} / \mathrm{l}$ & & \\
\hline Heptane & 365.9 & 48.1 & 98 & 4.3 & -3 & 0.684 & 0.037 & {$[20]$} \\
\hline
\end{tabular}




\begin{tabular}{|c|c|c|c|c|c|c|c|c|}
\hline Kerosene & 199 & 41.1 & $175-300$ & 0.04 & 52 & 0.80 & $\mathrm{n} / \mathrm{a}$ & {$[11][20]$} \\
\hline Methanol & 1180.7 & 22.8 & 64.6 & 4.6 & 12 & 0.791 & 0.008 & {$[20]$} \\
\hline
\end{tabular}

Table 2. Physical properties of fuels used in this study.

A uniform test procedure was followed for all experiments. First, the load cell with the pan and protective thermal insulation (protecting the load cell from excessive heat) was tared. Then the carpet was cut to the shape of the circular pan and fit into the pan. For the tests with sand, the carpet was substituted for by the filling of the pan with a specific amount of sand. The amount of sand used was random, as there is no direct comparability between different porous media. The total weight of the apparatus (fuel, porous media, pan, insulation) was within the measurement range of the load cell. The experimental setup is shown in Figure 4.

The initial weight of the carpet (or sand) and the pan was recorded. A certain amount of fuel was then spilled uniformly on the surface of the carpet. It was found that approximately 80 grams of fuel would cover the carpet in the pan, with no exposed rim and no extruding fibers. The fuel was allowed to sit in a quiescent environment for approximately 10 minutes while the fuel soaked uniformly into the carpet. Ignition was obtained with a butane igniter. Kerosene is difficult to ignite, as it has a fire point above the typical ambient temperature. Therefore, a paper wick served as a pilot flame in the center of the pool, providing the necessary pre-heating. During the test, the mass loss rate was recorded via the load cell and logged onto an A/D system.

\subsection{Non-Burning Evaporation}

Prior to the ignition, the heptane samples were allowed to evaporate for 10 minutes at ambient conditions. For a highly volatile fuel such as heptane, this was enough time to 
evaluate the evaporation rate. Due to the small variation in liquid level, the evaporation rate was very close to that of limited diffusion. The experiment was repeated for all three substrates (sand, water, carpet) and for different amounts of fuel. The evaporation rate of kerosene was almost negligible. To diminish the boundary (or edge) effects, a thin layer of water was used to minimize conduction from the pan. Water has a high thermal conductivity and has a relatively low thermal capacity. A water substrate was only used for pure heptane burning (i.e. the heptane floated on the water), as heptane has a low heat of evaporation and high heat of combustion. The water substrate was not monitored or controlled in this experiment. Heptane is more sensitive to the energy feedback than the other two fuels used in this study. Since the mass and heat transfer characteristics of carpet are much different than those of sand or water, determining the mass of water or sand to use in the reference tests was difficult to determine. Generally, the mass of sand or water was chosen to ensure that the fuel had a measurable evaporation rate while keeping the weight of the whole device within the measurement range of 500g. 


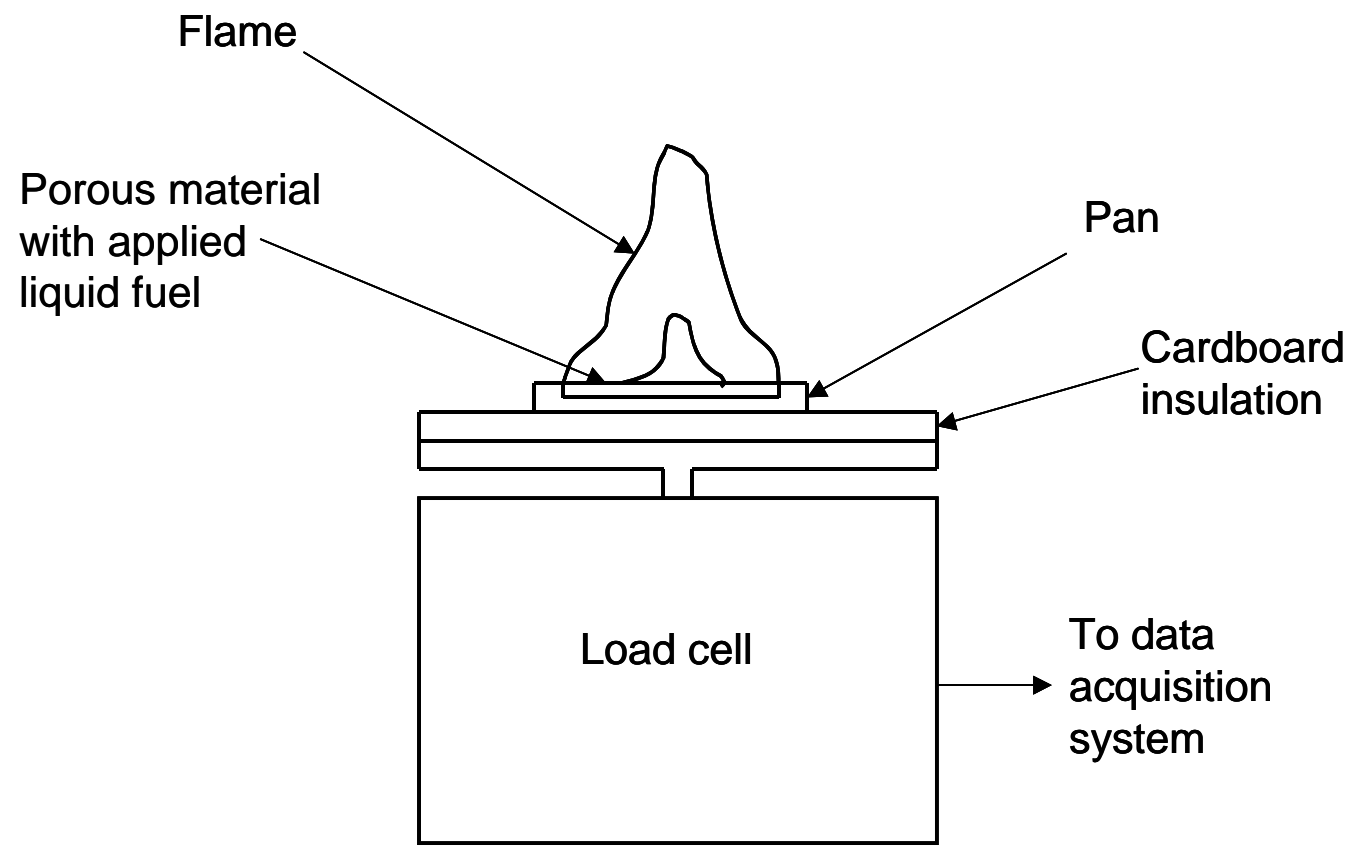

Figure 4. Experimental setup for determining evaporation rates from and burning rates of fuel/carpet specimens.

Figure 5 shows the average evaporation rate of heptane on different substrates. Due to the high vapor pressure of heptane, the evaporation process removes energy from the environment and the fuel beneath. Thus, heat transfer also dominates the evaporation process. It is evident that the evaporation rate of fuel on sand is smaller than that on water, since the larger conductivity of sand produces greater heat loss. Table 1 gives an order of magnitude estimate of the different thermal conductivities. The carpet has a much lower thermal conductivity than the water; therefore, it should have a lower evaporation rate from a heat transfer perspective. The absorbed energy from the environment acts only on the thin layer of fuel on carpet surface. But it is also shown that the evaporation rate of liquid on carpet is dependent on its initial mass, or in this analysis, the saturation level of fuel in the carpet. A decrease in saturation level at the carpet surface produced a lower evaporation rate. 


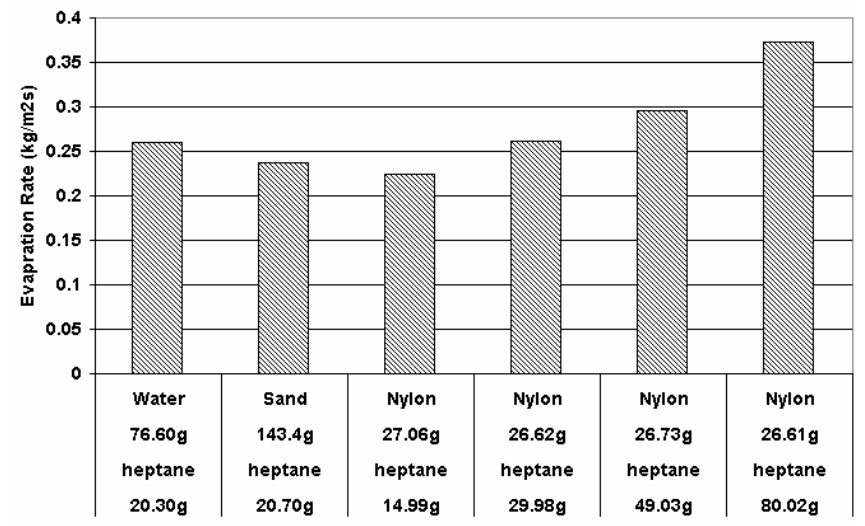

Figure 5. Evaporation rate of heptane on different substrates.

These results can also be explained using the concept of capillary motion. Under slow evaporation (compared with combustion), the capillary effect is more significant in supplying the fuel, so the total mass loss rate becomes dependent on the initial mass, as shown in Figure 5. Since evaporation and combustion share the same heat and mass transfer processes, the MBRR is found to be 1.43 by dividing the heptane evaporation rate on carpet with the heptane evaporation rate on water. This is less than that found by Hayasaka [11] (see Figure 3), but is comparable in magnitude.

\subsection{Combustion Experiments}

As discussed earlier, the combustion experiment is difficult to perform, since both the boundary effect and the capillary effect are difficult to isolate. Here, several small-scale tests, though primitive, can be explained in the framework of the above theory.

For the heat transfer dominated combustion, three different substrates were used and the results are presented in Figure 6. For heptane, the burning rate on sand is low in the beginning, but quickly increases to the free burning level. The sand conducts heat quickly to the boundary, but since the sand and the boundary have low heat capacity, their energy 
storage is low. Hence, the energy feedback returns the burning rate to the level of a freeburning pool. The burning rate of heptane on carpet is similar to the burning rate on water. This result is similar to the evaporation test (Figure 5). Here, the heat transfer and mass transfer processes both act on the mass-burning rate. With the carpet, the enhancement of the heat transfer is counter-acted by the limitation from the mass transfer process. So the total result is an insignificant change in the burning rate from that on water. Due to some uneven evaporation (the evaporation time was not strictly controlled) in the case of the heptane burning on sand, the total energy releases were not the same under the curves. The burning of the carpet fibers provides some additional energy into the total release. 

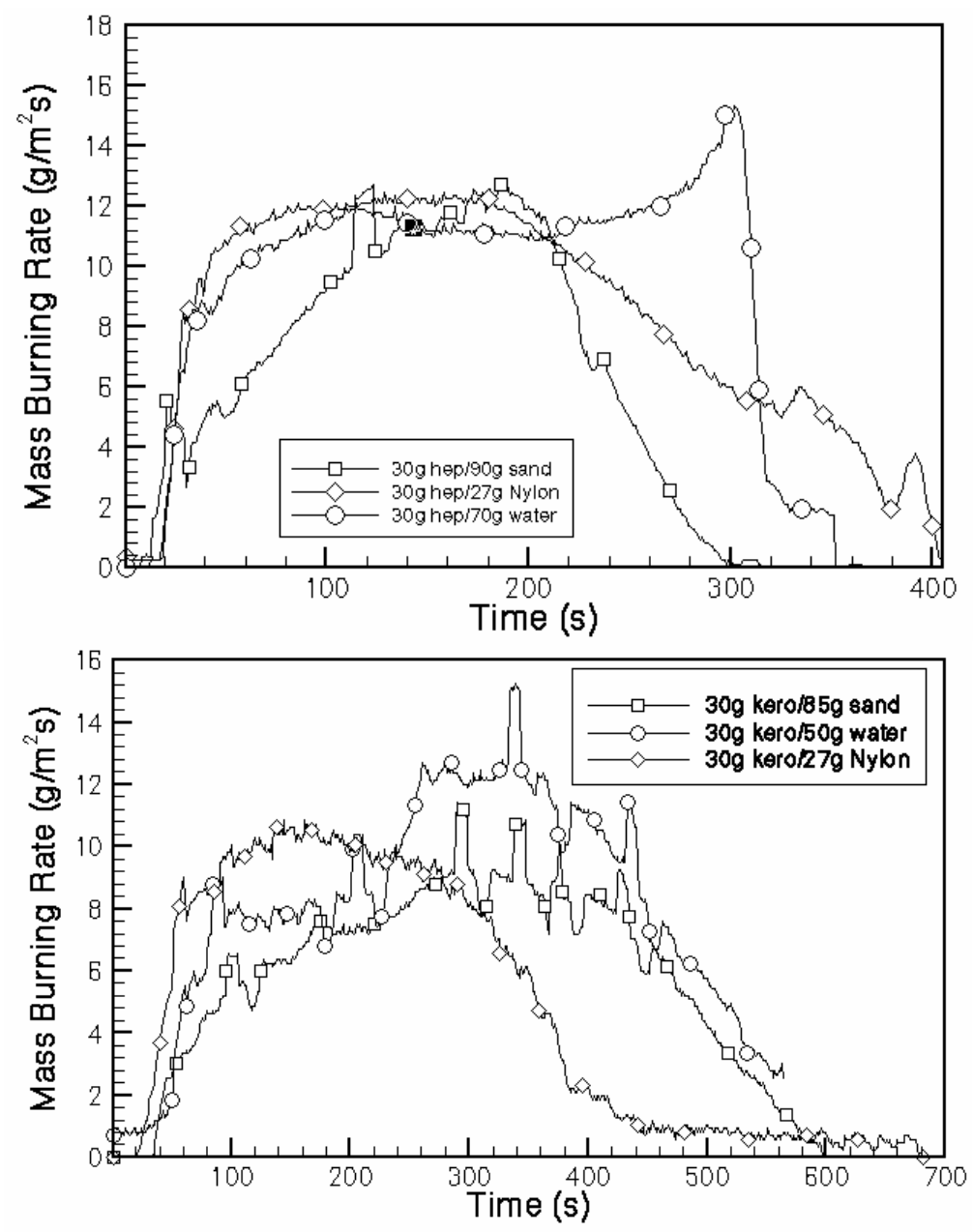

Figure 6. Heptane (up) and kerosene (down) burning rates on different porous media.

The kerosene combustion process revealed a similar result. But since the kerosene is not of uniform composition, the different components make the total combustion process unsteady. The strong soot level leads to a strong feedback to the fuel, part of which is lost and stored in the substrate and the boundary. When the heat capacity of the boundary is insufficient, that part of the energy is also returned to the burning process, producing a large burning rate for the late stage. For the early burning rate, the trend is same as that of heptane. Here the water served as insulation material that may contribute to the massburning rate, but only in the later stage when there is not enough liquid fuel evaporation for cooling. 

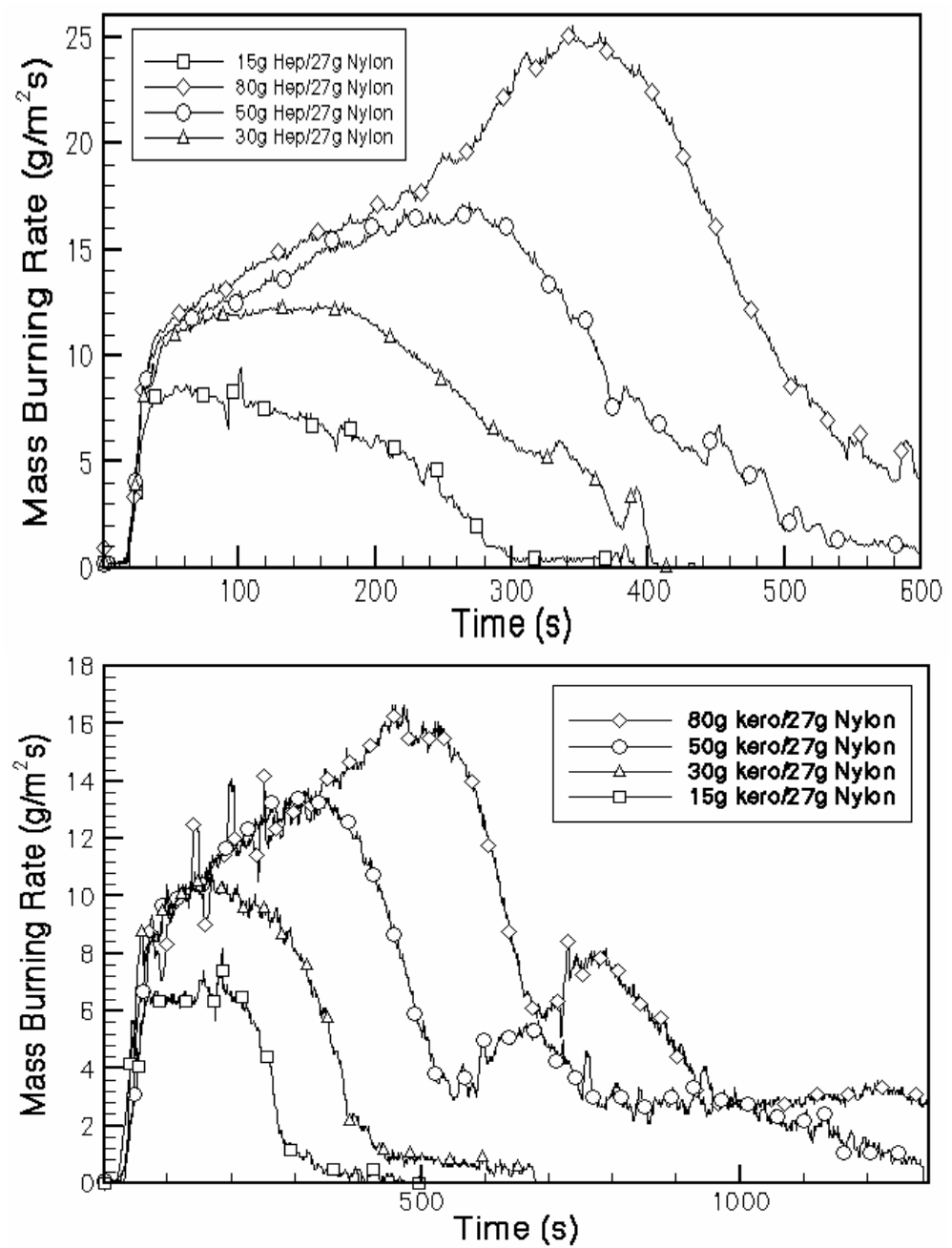

Figure 7. Mass-burning rates of heptane (left) and kerosene (right) burning on carpet for different amounts of initial fuel.

For the mass transfer limited combustion, different amounts of fuel were spilled on the carpet (Figure 7). Generally, the maximum amount of fuel the carpet can hold is determined by the thickness of the carpet [19]. For small amount of fuel, the burning rate curve has two stages, the steady burning and a decaying period, which is supported by the findings of Wentworth and Torero [18]. For a large amount of fuel, the interaction of the fuel with the boundary becomes significant, as the boundary cannot maintain the rate of the heat loss from beginning to end. This leads to a constantly increasing mass-burning rate, until the majority of the fuel is consumed. The maximum burning rate of heptane 
was almost double that of the normal free-burning pool fire, well above the MBRR prediction (1.6 in Figure 3). One possible explanation for this behavior may be the role of boundary conduction on the energy balance. Heptane has a low flash point, low heat of evaporation, and the fire is sooty, hence the fuel will readily react to heat transfer from the boundary. Babrauskas et al. [23] found that the edge of the sample holder will lower the peak release rate, but their samples are mostly solids, which is insensitive to the boundary temperature. The boundary will lose some energy at first; however, as the walls of the pan heat up, the boundary conduction will enhance the mass-burning rate. Seeger [24] measured the heat loss through the wall boundary term, and his conclusions are similar. Both studies were based on the heat transfer in the early stages of fire growth. At the later stages, when all the fuel was heated to its boiling temperature, the heat capacity of the boundary has an enhanced role. The energy conducted from the hot wall will enhance the evaporation of more fuel.

Another possible explanation is that the strong heat transfer makes the thermal layer thinner, and since the carpet fiber is immersed in the fire, the extended surface enhanced the mass-burning rate. To fully understand the interaction between the boundary and the fuel, more experimental work is still needed.

Kerosene is a mixture of various hydrocarbons; hence, its MBRR cannot be calculated from any physical properties. In this study, a value of 1.3 was measured in the experiment, which is close to Hayasaka’s measurement (Figure 3). 
As shown in Figure 7, if enough fuel is supplied, the largest mass-burning rate for the fuel on the carpet is significantly larger than the burning rate of fuel floating on water. With relatively little fuel in the carpet, the mass-burning rate is lower than the fuel on water burning rate. After experiencing a peak, which comes from the feedback boundary, the burning rate decays, which is a combination of both fuel supply and the mass transfer limitation (not enough capillary effects).

A closer inspection of the MBRR is warranted. MBRR is defined as the ratio between the maximum possible burning rate and the minimum possible burning rate. The maximum value of the MBRR is determined by the energy balance. The real burning rate is always lower than the maximum burning rate, with the deficit dependant on the amount of energy needed in the evaporation process. If the inverse of the MBRR is considered, this provides a measure of the energy required for direct evaporation. The existence of the boiling zone in Hayasaka's experiment or the carpet in this study provides a mechanism to decrease the heat loss term and thus return the mass-burning rate closer to its ideal value.

Sustained fuel burning on carpet provides a heat flux that allows the carpet backing material (which is comprised primarily of dense polyurethane) to pyrolyse and/or burn. This leads to a second peak in mass-burning rate history (Figure 7). The residues of different amounts of kerosene burning on carpet are shown in Figure 8. 


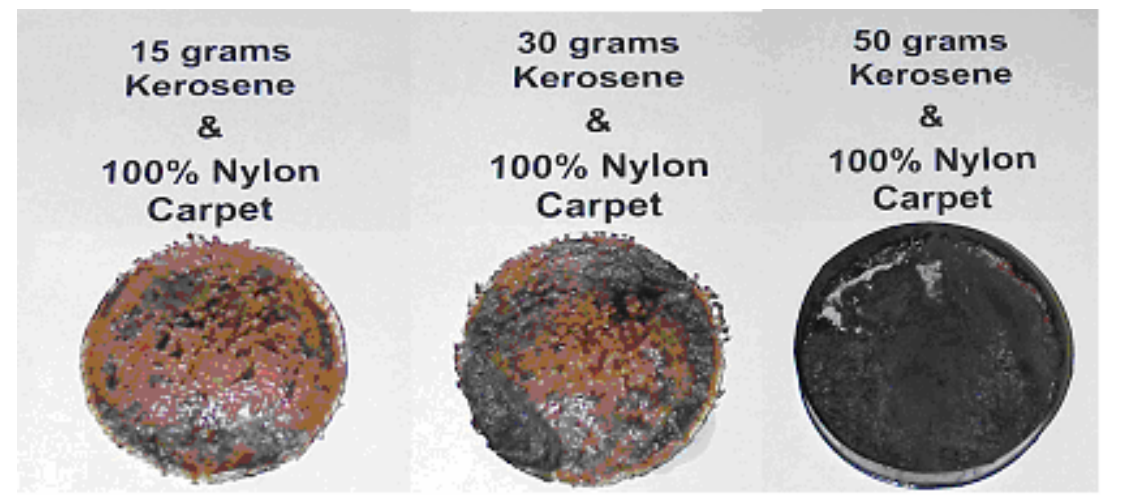

Figure 8. Residue left by different amounts of fuel burning on carpet.

\subsection{Maximum burning rate ratio}

An additional experiment was performed with methanol used to soak the carpet. Compared with both heptane and kerosene, the methanol molecule has only one carbon atom, which means low soot in the flame and less radiative feedback to the fuel. The methanol also has a large heat capacity, so the total behavior of burning methanol was rather insensitive to the boundary condition. The burning rate of methanol was thus characterized by an almost constant value in time (Figure 9). 80g of methanol was enough to flood the carpet to the fiber tip. Generally, the methanol burning on carpet was divided into two stages, steady burning and decay burning. In the steady burning stage, the mass-burning rate of methanol on carpet was enhanced by a factor of $10 \%$, while the decay stage occurs earlier than for the free burning pool fire. The total area under the curve (total energy release) is almost the same. For the free burning pool fire, the decay period was short, due to insufficient fuel. But for methanol burning on carpet, the fuel supply is gradually decreased, due to a decreasing saturation level in carpet and due to insufficient capillary effects. 


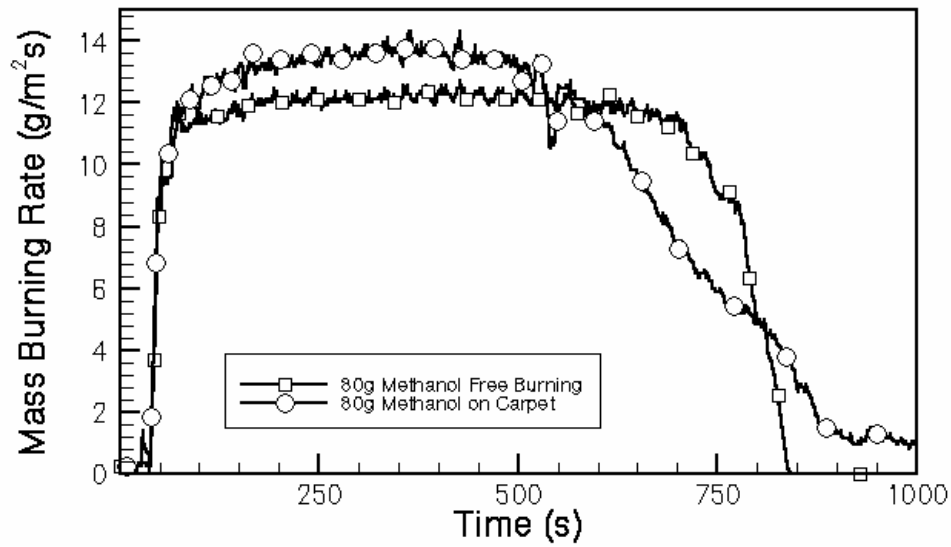

Figure 9. Methanol burning with and without carpet

By averaging the steady burning rate, the enhancement was about $10 \%$, which is close to the prediction in Figure 3. Due to the large latent heat of methanol, Hayasaka [11] was not able to find the difference for methanol burning. Here, a different heat transfer blocking mechanism was applied and the theoretical prediction value is achieved. This is a very good indication of the validity of the MBRR concept.

\section{Further Discussions}

\subsection{Capillary or wick effects}

As a fuel supply mode, the capillary effect is difficult to characterize. DeHaan [19] noticed the enhancement of pentane evaporation due to the presence of the carpet, but he explained that phenomenon alone through the capillary action due to the suction potential. However, this interpretation seems incomplete, since DeHaan [19] did not make clear the relationship between heat and mass transfer and the role of capillary effects. Here the capillary effects still could not be defined, but the analysis is based on the fact that the capillary action is enough to supply the fuel. The capillary effect cannot enhance the mass-burning rate beyond its maximum value, which is set by the energy balance. 
It is true that the evaporation area is extended by the carpet fibers, but the effective area for diffusion (same as pan area) is almost the same, and the capillary effect itself can not increase the fuel vapor concentration ( $\rho_{c g \Delta}$, see Figure 2 ) above $100 \%$. Only in the later stages, when the fuel level is low, do the capillary effects become significant. Without the capillary effect, the surface temperature will be high enough to ignite the fibers, which will not happen for saturated carpets in the early stage. For some other porous media, such as glass beads or sand, the capillary effect may be insufficient to supply the fuel, and the liquid fire will die out gradually without sufficient fuel supply [15].

From the above analysis, the capillary effect itself will not enhance the mass-burning rate of the liquid fire burning on carpets. The capillary effect is significant only when the fuel supply is insufficient, which lowers the mass-burning rate. This behavior was also shown in Wentworth and Torero's experiments [18]. This may not be an important issue for fire investigation.

\subsection{Evaporation, combustion and heat transfer}

Several other researchers have contributed to the heat transfer analysis of the pool fire. As noted above, DeHaan [19] did significant work on the liquid evaporation on carpets. DeHann attributed the burning rate enhancement to the capillary rise; however, this phenomenon is difficult to characterize. DeHaan [19] used highly evaporative liquids in his experiments. The fire point of these fuels is typically below ambient temperature; hence the evaporation rate is readily measured. Putorti et al. [21] conducted an extensive 
study of burn patterns and found that the peak heat release rate for the fuel spills on nonporous surfaces (cement, wood floor, etc.) are approximately a quarter to an eighth of the heat release rates of equivalent diameter pool fires burning over deep liquid pools. Due to the free spread of spilled liquid, the fuel is thin on non-porous ground and the burning duration is short. The conductivity of the floor (cement, wood, etc.) is much larger than that of the fuel, resulting in a greater heat loss due to conduction to the base material. Putorti and co-workers found that the peak heat release rates of fuel burning on carpets are approximately equal to the steady state heat release rates of the equivalent diameter pool fires. This finding is not completely in agreement with the present results, however, since the amount of fuel poured on the carpet was not specified, it is possible to achieve that result, depending on the level of fuel in the carpet (see Figure 7). From their qualitative experiments, it is still difficult to assess if the comparable magnitudes are just coincidental.

The results of Putorti et al. [21] also indicate that the inverse of MBRR is a good concept to describe the heat loss condition for the liquid fuel to burn. The concept of MBRR is based on the mass-burning rate of early burning with in-depth heat loss, which is transient and difficult to characterize. The maximum burning rate or steady burning rate is a constant set by the energy balance, so the inverse of MBRR can better describe the contributions from the boundary condition and other loss terms. To better understand the role of the backing material, the mass-burning rates of different fuels on different surfaces should determined. 


\subsection{Mass transfer limited combustion}

Experimental data presented by Blackshear and Kanury [16] presents a different scenario to that studied here. Blackshear and Kanury conducted experiments using a porous matrix with a similar thermal conductivity to the fuel and showed that the burning rate with and without a substrate remained invariant. Again, since there is no information about the saturation level of the liquid in porous media, their findings are not in conflict with our result. Tao and Kaviany [17] reported a decreased mass-burning rate with their ceramic burner as porous medium, having a conductivity comparable to that of the fuel, so they could disregard the heat transfer effect. Wentworth and Torero [18] found two stages of liquid burning on insulation material: steady burning and decay burning. The decaying burning period is close to the mass transfer limited combustion process. Those studies focused on the mass transfer side of combustion phenomena, while liquid burning on carpet is a combination of the two processes.

In this study the carpet serves two conflicting roles. As an insulating material, the carpet increases the mass-burning rate, by keeping the fuel temperature high. As a porous medium, it decreases the burning rate due to the limitation from a mass transfer perspective, when the capillary effect is insufficient. This conflict lessens the overall effect of either process.

\section{Conclusions}

The seemingly simple scenario of a liquid fuel burning on carpet is in fact a very complex situation. Boundary conditions, fuel level and fuel properties can play an important role 
on the behavior of such a fire. In this study, heat transfer and mass transfer theory was applied in the analysis of this problem.

The capillary effect of fuel rising along the fiber does have an important role in supplying the fuel to the fire. Some researchers have indicated that the capillary effect will enhance the mass-burning rate. However, this should be accomplished with good ventilation or in the forced air flow. Here the enhancement to mass transfer by the extended surfaces has a minor contribution to total mass burning rate. Instead, the energy balance dominates the process.

While most dry porous media (e.g. sand) have the same effective diffusion area as the surface of a free-burning pool fire, the presence of the media will reduce the mass transfer rate. However with carpet, the capillary forces compensate for the limitation from diffusion by drawing the fuel to the fiber tip. Thus, the reduction is not significant. Only when the fuel level is low will the mass-burning rate be decreased as a result of an insufficient capillary effect. The global result is that the variance in the mass transfer in the early stage can be ignored.

The presence of carpet serves primarily as an insulation material, which blocks the heat loss to the depth of the pool, thus enhancing the mass-burning rate. Moreover, the boiling zone on the surface of the carpet fiber is also insulating the energy transfer, as the heat cannot be transferred to the incoming fuel supplied by capillary motion, so the temperature gradient happens almost on the surface of carpet fiber. Thus, an increased 
burning rate is achieved by minimizing the loss term. The concept of MBRR is a useful concept to quantify this enhancement.

The enhancement of the burning rate does not occur in all stages of burning. The carpet enhances the mass-burning rate from its earlier value with in-depth heat loss to a higher rate without heat loss. The carpet serves as an insulation material, making the spilled liquid burning behave more like deep pool steady burning. This is the root cause of some observations that liquid fires are more severe on carpet than on normal ground.

As is commonly known, the boundary conditions play an important role in the massburning rate of the fuel burning in pool fire mode. The MBRR concept highlights this by providing the ratio of the maximum mass burning rte to the minimum mass burning rate. The minimum mass burning rate provides the energy necessary to evaporate the fuel. Though the theoretical MBRR is helpful to show the quantitative enhancement due to the carpet, the inverse of MBRR shows the loss fraction due to the boundary conditions. This is important and a better concept in defining the proper energy release rates for initial fire development. The initial energy release rate of an incendiary fire should be carefully chosen for fire modeling tools, such as FPETOOL or FDS (Fire Dynamics Simulator).

The above analysis is valid for pool fires of intermediate size, (i.e., 5-20 cm in diameter), when convection is the dominant mode of heat transfer. This is an important dimension of liquid pool fires in incendiary fires, where the fuel amount is limited. With pool fires of larger diameter, radiation plays a dominant role, and the assumption of a constant heat 
feedback will eventually breakdown. These fires will require a more detailed analysis of the evolution of the heat feedback. To our knowledge, no experiments have been performed on large-scale liquid fuel fires on porous media.

\section{Acknowledgements}

The authors would like to thank David Wilmot for his help during the experiments. 


\section{References}

[1] Benjamin, I.A., Davis, S., Flammability Testing for Carpet, Fire Technology, Vol.15, No. 3. Aug 1979, 189-194

[2] Roby, R., Carpenter, D.J., The Role of Carpet in Determining Origin and Cause of a Compartment Fire, Fire and Arson Investigator, October 1999.

[3] Joulain, P., The Behavior of Pool Fires: State of the art and new Insights, 27th Symposium on Combustion, 1998, 2691-2706

[4] Blinov, V.L., Khudiakov, G.H., Certain Laws Governing Diffusive Burning of Liquids, Accademmiia Nauk, SSSR Doklady 113, 1957, 1094-1098.

[5] Hottel, M., Certain Laws Governing Diffusive Burning of Liquids, Fire Research Abstracts and Reviews, 2, 1958, 41-44.

[6] Spalding, D.B., The Combustion of Liquid Fuels, 4th Symposium on Combustion, 1953, 847-864.

[7] Burgess, D.S., Strasser, A., Grumer, J., Diffusive Burning of Liquid Fuels in Open Trays, Fire Research Abstracts and Reviews 3,1961, 177-192.

[8] Akita, K., Some Problems of Flame Spread Along a Liquid Surface, 14th Symposium on Combustion, 1973, 1075-1082.

[9] Klassen, M., Gore, J.P., Sivathanu, Y.R., Radiative Heat Feedback in a Toluene Pool Fire, 24th Symposium on Combustion, 1992, 1713-1719.

[10] Hamins, A., Yang, J.C., Kashiwagi, T., A Global Model for Predicting the Burning Rates of Liquid Pool Fires, NISTIR6381, 1999.

[11] Hayasaka, H., Unsteady Burning Rates of Small Pool Fires, 5th Symposium on Fire Safety Science, 1997.

[12] Ishida, H., Flame Spread over Fuel-soaked Ground, Fire Safety Journal, 10, 1986, 163-171.

[13] Takeno, K., Hirano, T., Flame Spread over Porous Solids Soaked with a Combustible Liquid, 21st Symposium on Combustion, 1986, 75-81.

[14] Takeuchi, T., Tsuruda, T., Ishizuka, S., Hirano, T., Burning Characteristics of a Combustible Liquid Soaked in Porous Beds, 3rd Symposium on Fire Safety Science, Edinburgh, Scotland, July 1991.

[15] Suzuki, T., Hirano, T., Effects of Gasification Properties of a combustible on Flame Spread over its Surface, 3rd Symposium on Fire Safety Science, Edinburgh, Scotland, July 1991.

[16] Blackshear, P.L. Jr., Kanury, M.A., Heat and Mass Transfer to, from and within Cellulosic Solids Burning in Air, 10th Symposium on Combustion, 1965, 811.

[17] Tao, Y.X., Kaviany, M., Burning Rate of Liquid Supplied Through a Wick, Combustion and Flame, 86, 1991, 47-61.

[18] Wentworth, B.T., Torero, J.L., The Effect of External Radiation and a Porous Media on Ignition and Mass Transfer of a Liquid Fuel, Proceedings of NHTC’01, June 10-12, Anaheim, California, 2001.

[19] DeHaan, J.D., The Reconstruction of Fires Involving Highly Flammable Hydrocarbon Liquids, $\mathrm{PhD}$ Thesis, University of Strathclyde, Glasgow, 1995

[20] http://webbook.nist.gov/chemistry/, NIST website for material properties.

[21] Putorti, A., McElroy, J.A., Madrzykowski, D., Flammable and Combustible Liquid Spill/Burn Patterns, NIJ Report 604-00, 2000.

[22] SFPE Handbook of Fire Protection Engineering, $2^{\text {nd }}$ edition, 1995

[23] Babrauskas, V.; Twilley, W. H.; Parker, W. J., Effects of Specimen Edge Conditions on Heat Release Rate. Fire and Materials, Vol. 17, No. 2, 51-63, March/April 1993.

[24] Seeger, P.G., On the combustion and heat transfer in fires of liquid fuels in tanks, Heat tranfer in fires, edited by P.L. Blackshear, 1974 